The $Q_{\text {weak }}$ Experiment at Jefferson LabA Search for New Physics at the TeV Scale

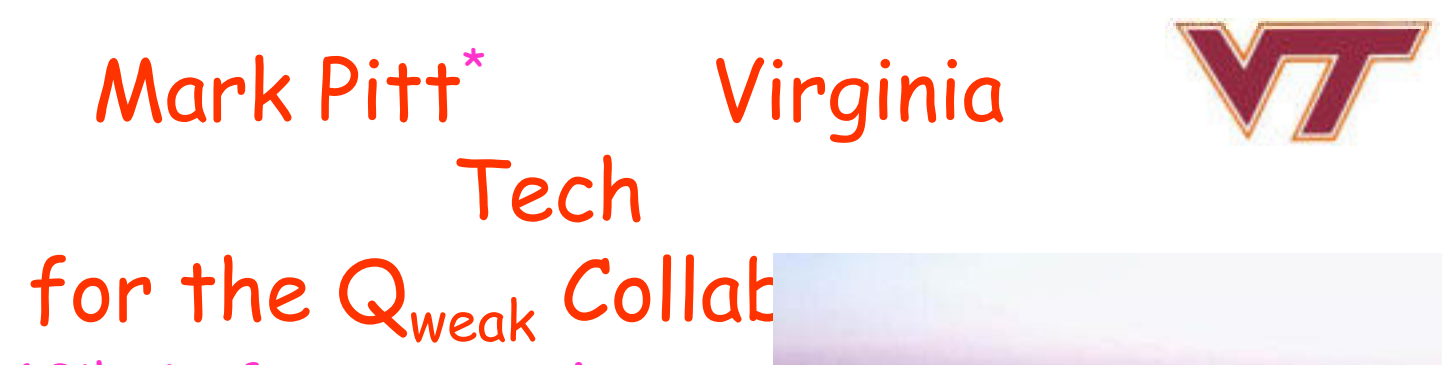

CIPANP 2009: 10 th Conference on the Intersections of Particle and Nuclear Physics

La Jolla, CA May 26-31, 2009
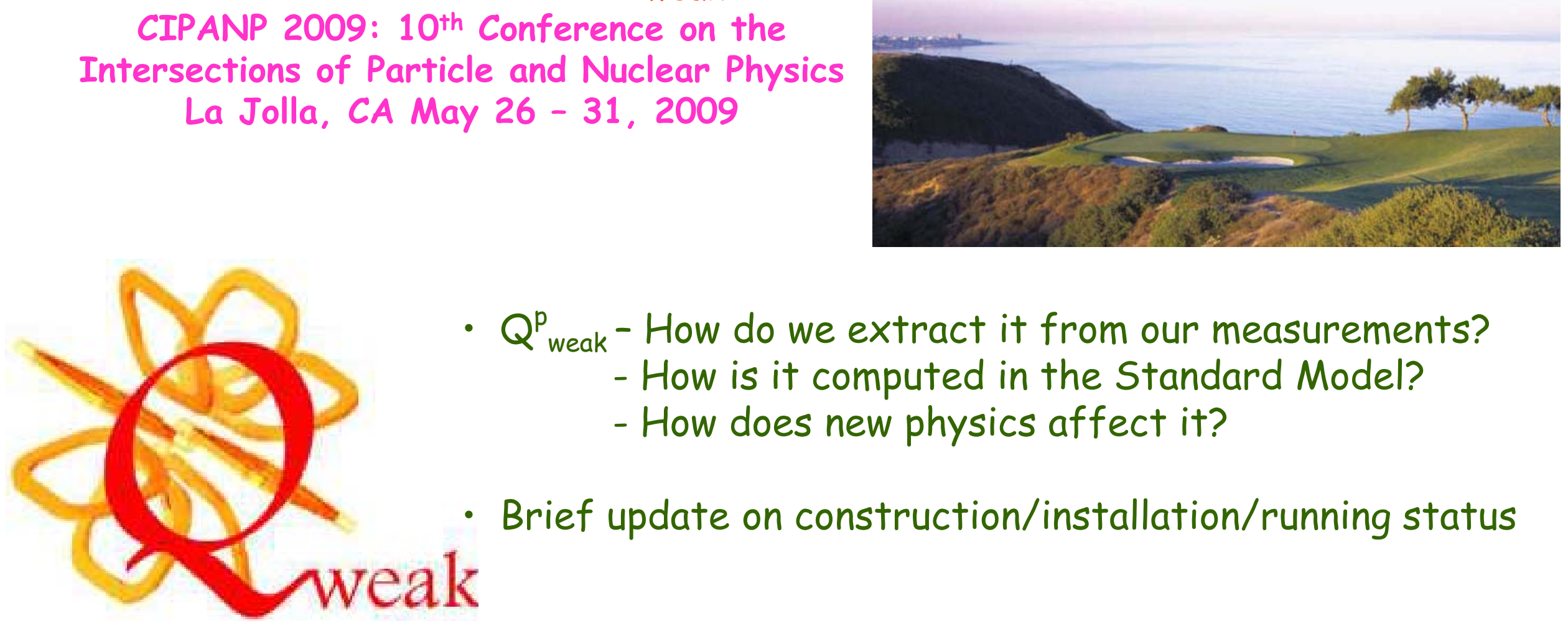

- $Q_{\text {weak }}^{p}$ - How do we extract it from our measurements?

- How is it computed in the Standard Model?

- How does new physics affect it?

- Brief update on construction/installation/running status

* Work partially supported by the National Science Foundation 


\section{The Qweak Collaboration}
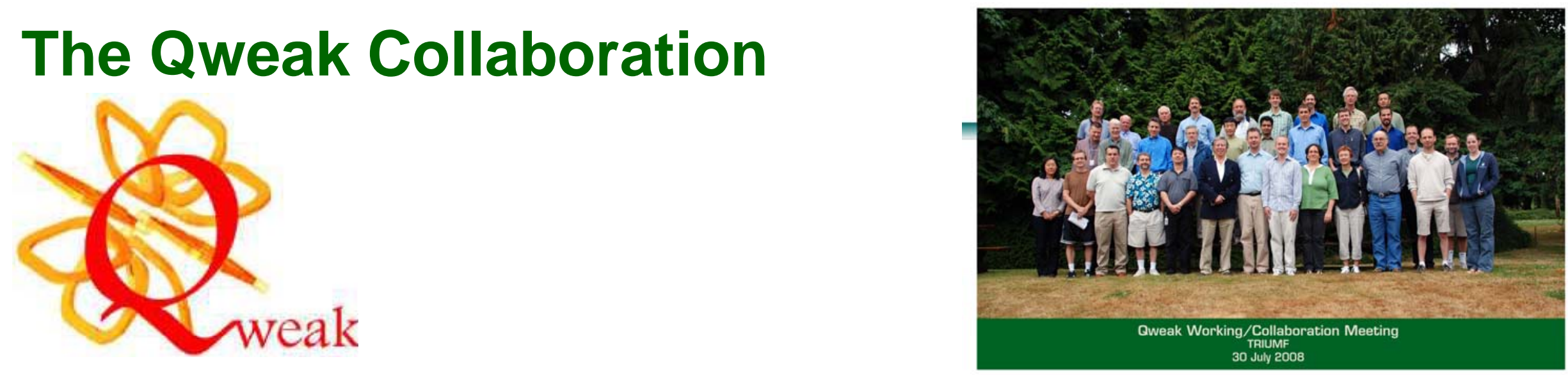

D. Armstrong, A. Asaturyan, T. Averett, J. Benesch, J. Birchall, P. Bosted, A. Bruell, C. Capuano,

R. D. Carlini ${ }^{1}$ (Principal Investigator), G. Cates, C. Carrigee, S. Chattopadhyay, S. Covrig, C. A. Davis,

K. Dow, J. Dunne, D. Dutta, R. Ent, J. Erler, W. Falk, H. Fenker, T. A. Forest, W. Franklin,

D. Gaskell, M. Gericke, J. Grames, K. Grimm, F.W. Hersman, D. Higinbotham, M. Holtrop,

J.R. Hoskins, K. Johnston, E. Ihloff, M. Jones, R. Jones, K. Joo, J. Kelsey, C. Keppel, M. Khol, P. King, E. Korkmaz, S. Kowalski ${ }^{1}$, J. Leacock, J.P. Leckey, L. Lee, A. Lung, D. Mack, S. Majewski, J. Mammei, J. Martin, D. Meekins, A. Micherdzinska, A. Mkrtchyan, H. Mkrtchyan, N. Morgan, K. E. Myers, A. Narayan, A. K. Opper, SA Page ${ }^{1}$, J. Pan, K. Paschke, M. Pitt, M. Poelker, T. Porcelli, Y. Prok, W. D. Ramsay, M. Ramsey-Musolf, J. Roche, N. Simicevic, G. Smith², T. Smith, P. Souder, D. Spayde, B. E. Stokes, R. Suleiman, V. Tadevosyan, E. Tsentalovich, W.T.H. van Oers, W. Vulcan, P. Wang, S. Wells, S. A. Wood, S.

Yang, R. Young, H. Zhu, C. Zorn

\section{${ }^{1}$ Spokespersons 2 Project Manager}

College of William and Mary, University of Connecticut, Instituto de Fisica, Universidad Nacional Autonoma de Mexico, University of Wisconsin, Hendrex College, Louisiana Tech University, University of Manitoba, Massachusetts Institute of Technology, Thomas Jefferson National Accelerator Facility, Virginia Polytechnic Institute \& State University, TRIUMF, University of New Hampshire, Yerevan Physics Institute, Mississippi State University, University of Northern British

Columbia, Cockroft Institute of Accelerator Science and Technology, Ohio University, Hampton University,

University of Winnipeg, University of Virginia, George Washington University, Syracuse University,

Idaho State University, University of Connecticut, Christopher Newport University 


\section{The $Q_{\text {weak }}^{p}$ Experiment: A Search for New TeV Scale Physics via a Measurement of the Proton's Weak Charge}

\begin{tabular}{|l} 
Measure: Parity-violating asymmetry in \\
$\qquad \begin{aligned} \vec{e}+p \text { elastic scattering at } Q^{2} \sim 0.03 \mathrm{GeV}^{2} \\
\text { to } \sim 4 \% \text { relative accuracy at JLab }\end{aligned}$ \\
Extract: Proton's weak charge $Q^{p}$ weak $\sim 1-4 \sin ^{2} \theta_{W}$ \\
to get $\sim 0.3 \%$ on $\sin ^{2} \theta_{W}$ at $Q^{2} \sim 0.03 \mathrm{GeV}^{2}$ \\
\\
tests "running of $\sin ^{2} \theta_{W}$ " from $\mathrm{M}^{2} z$ to low $Q^{2}$ \\
sensitive to new TeV scale physics
\end{tabular}


"Running of $\sin ^{2} \theta_{w}$ " in the Electroweak Standard Model

- Electroweak radiative corrections

$\rightarrow \sin ^{2} \theta_{W}$ varies with $Q$
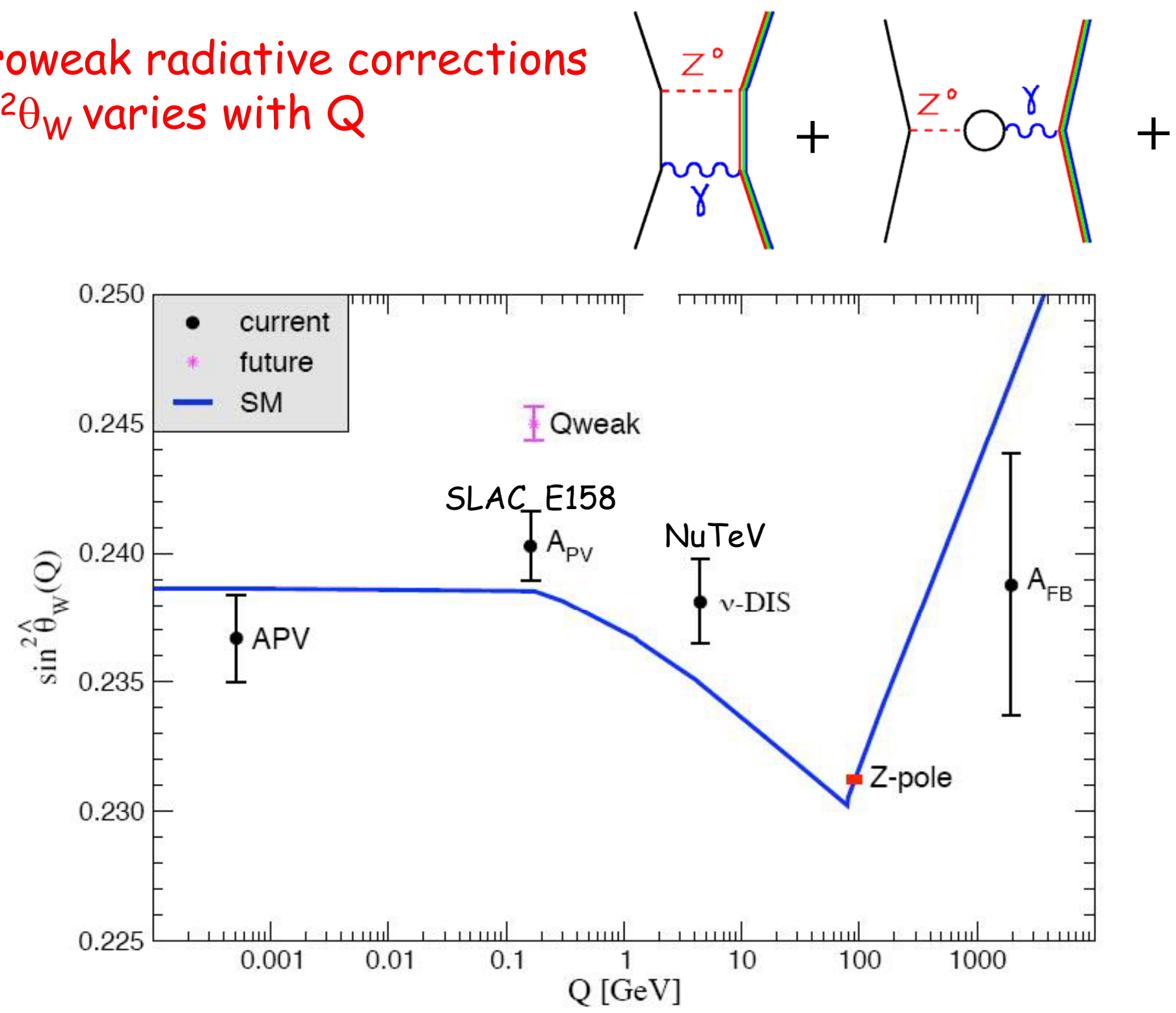

PDG 2008 Review: "Electroweak Model and Constraints on New Physics"

J. Erler \& P. Langacker 
Neutral Current Observable - Parity-Violating Asymmetry

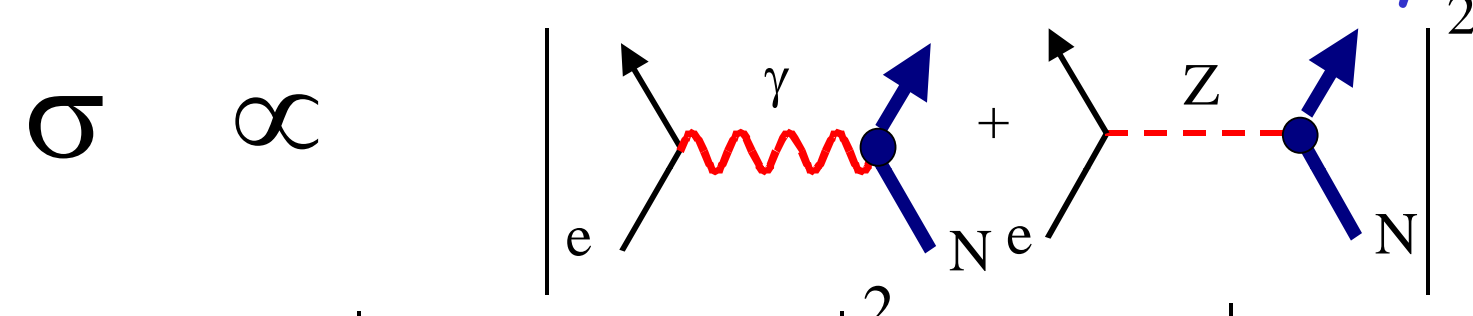

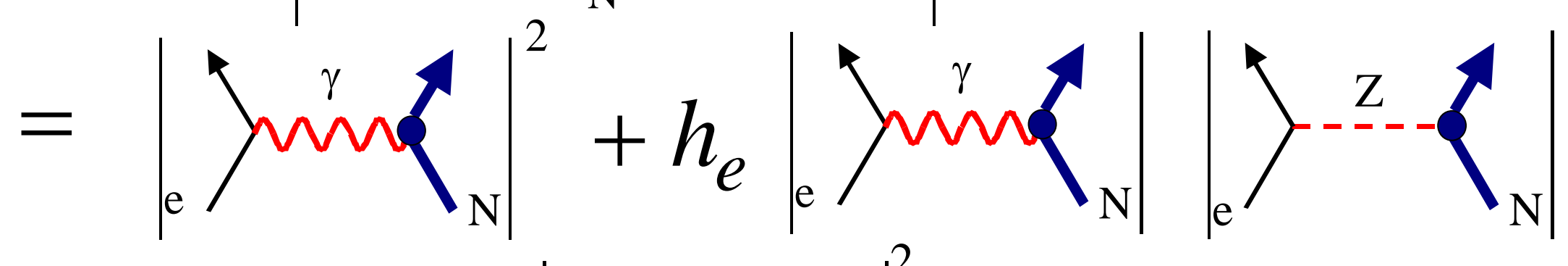

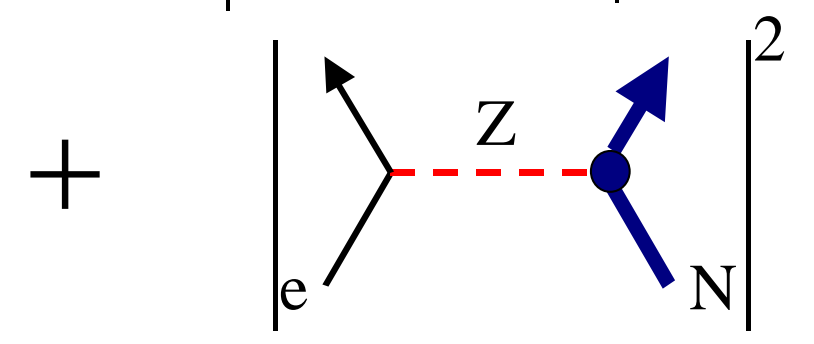

$\overrightarrow{\mathrm{e}}+\mathrm{N}$

(elastic scattering)

$$
\begin{aligned}
A & =\frac{\sigma_{R}-\sigma_{L}}{\sigma_{R}+\sigma_{L}} \propto \frac{\text { e }}{\mid\left\langle r v q_{p \mathrm{e}}|\cdots|_{\mathrm{p}}\right.} \\
& =\left[\frac{-\mathrm{G}_{\mathrm{F}} Q^{2}}{4 \pi \alpha \sqrt{2}}\right] \times F\left[\text { form factors, } \sin ^{2} \theta_{W}\right] \approx 3 \times 10^{-7}=0.3 \mathrm{ppm}
\end{aligned}
$$




\section{$Q^{p}{ }_{\text {weak }}:$ Extract from Parity-Violating Electron Scattering}

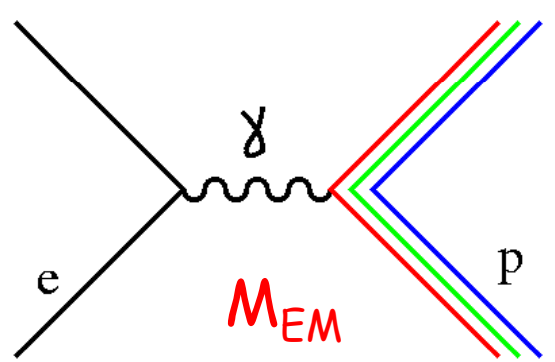

measures $Q^{p}$ - proton's electric charge

$$
\text { As } Q^{2} \rightarrow 0
$$

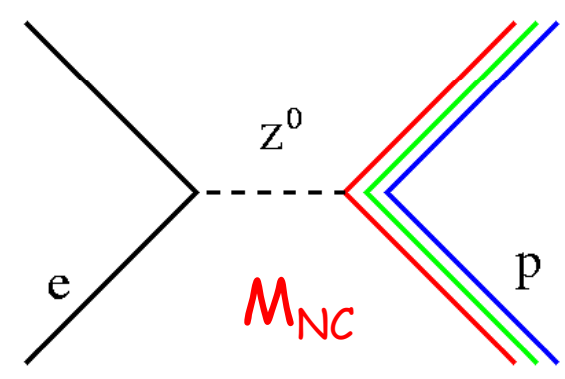

measures $Q^{P_{\text {weak }}}$ - proton's weak charge

$$
\begin{aligned}
A=\frac{2 M_{N C}}{M_{E M}}= & {\left[\frac{-G_{F}}{4 \pi \alpha \sqrt{2}}\right]\left[\mathrm{Q}^{2} \mathrm{Q}_{\text {weak }}^{p}+F^{p}\left(\mathrm{Q}^{2}, \theta\right)\right] } \\
\stackrel{\substack{\mathrm{Q}^{2} \rightarrow 0 \\
\theta \rightarrow 0}}{\longrightarrow} & {\left[\frac{-G_{F}}{4 \pi \alpha \sqrt{2}}\right]\left[\mathrm{Q}^{2} \mathrm{Q}_{\text {weak }}^{p}+\mathrm{Q}^{4} B\left(Q^{2}\right)\right] } \\
\mathrm{Q}_{\text {weak }}^{p} & =1-4 \sin ^{2} \theta_{W} \sim 0.072 \text { (at tree level) }
\end{aligned}
$$

- $\mathrm{Q}_{\text {weak }}$ is a well-defined experimental observable

- $Q^{p}{ }_{\text {weak }}$ has a definite prediction in the electroweak Standard Model

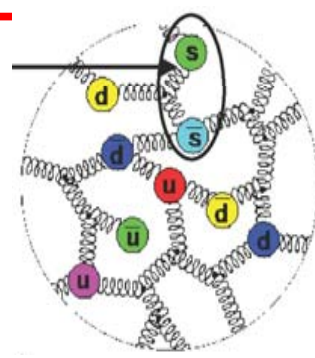

$$
\begin{array}{r}
G_{E}^{s}\left(Q^{2}\right) \\
G_{M}^{s}\left(Q^{2}\right)
\end{array}
$$

Strange electric and magnetic form factors -measure contribution of strange quark sea to nucleon structure 


\section{Nucleon Structure Contributions to the Asymmetry}
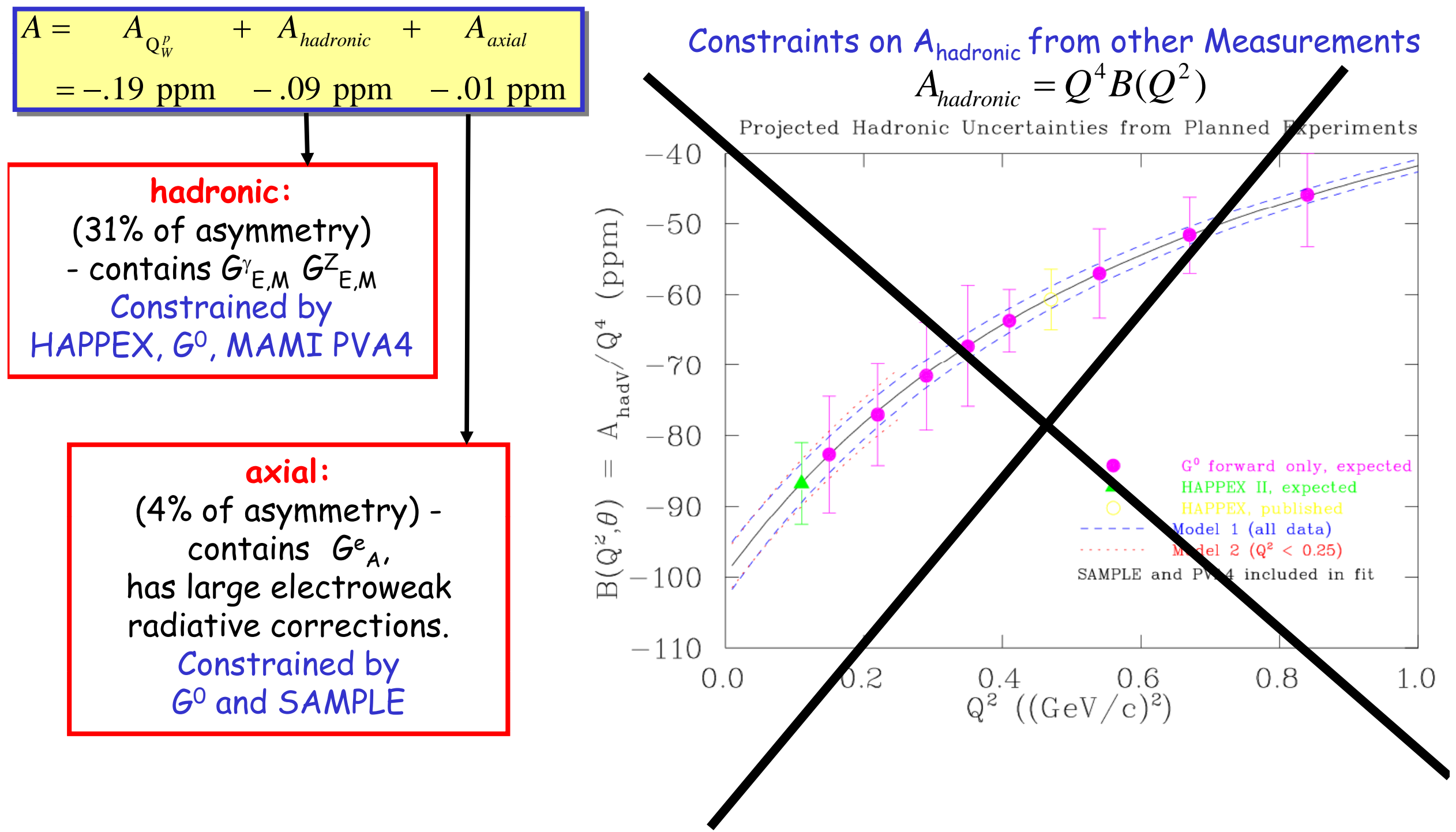


\section{Strange Form Factor Experiments - Now There's Data!}

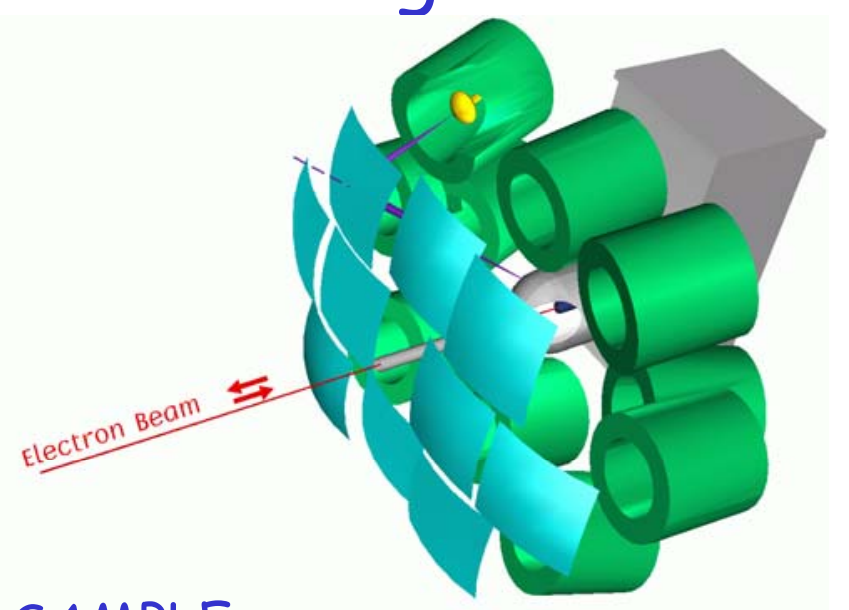

SAMPLE:

Location: MIT-Bates

Targets: $p, d$

Kinematics: backward angle, $Q^{2}=.038,10 \mathrm{GeV}^{2}$

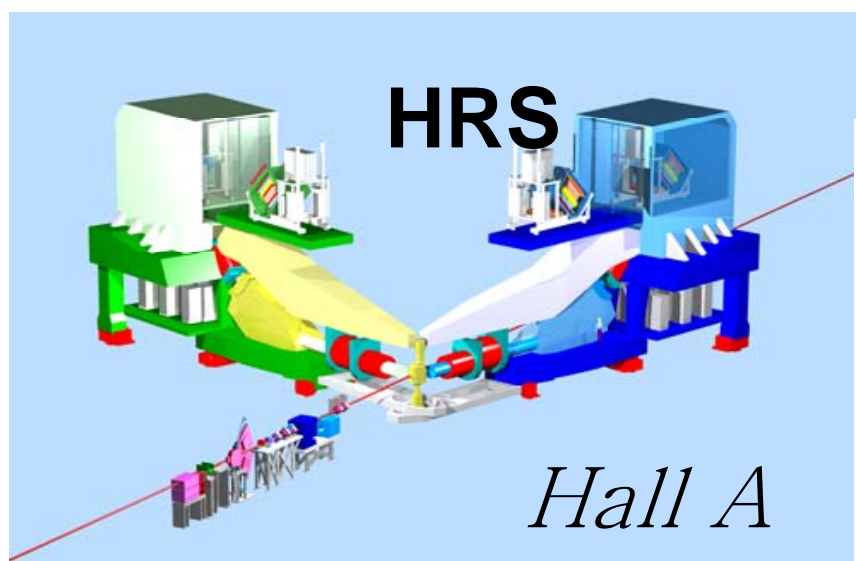

HAPPEX I, II, III:

Location: Jefferson Lab Hall A

Targets: $p,{ }^{4} \mathrm{He}$

Kinematics: forward angle, $Q^{2}=.10, .48 \mathrm{GeV}^{2}$
See Nuclear and

Nucleon Structure -9 tomorrow

(Thomas, Souder, Beise)

Mainz PV-A4:

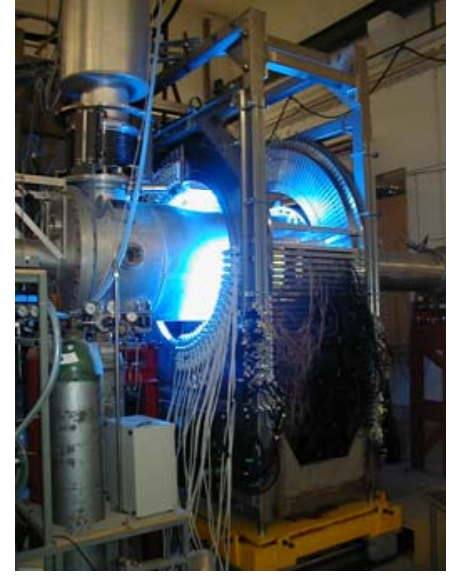

Location: Mainz MAMI microtron

Targets: $p, d$

Kinematics: forward \& backward angles $Q^{2}=.11, .23 \mathrm{GeV}^{2}$

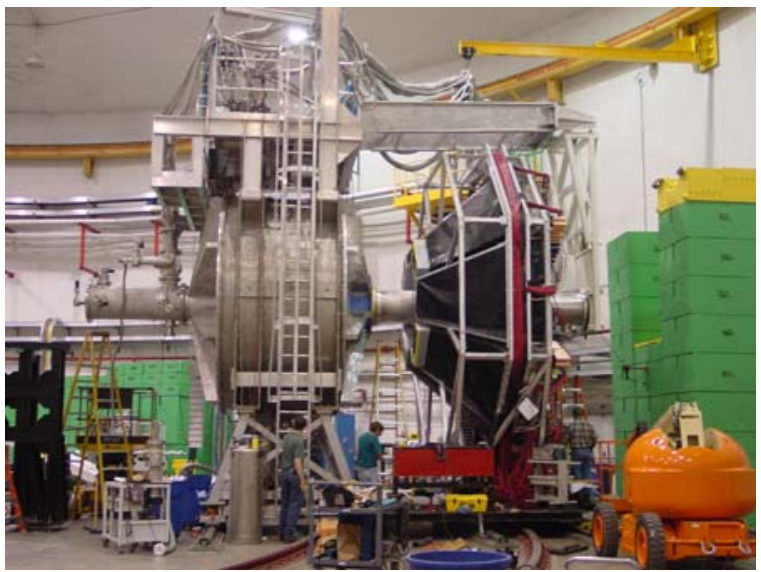

G:

Location: Jefferson Lab Hall C

Targets: $p, d$

Kinematics: forward \& backward angles $Q^{2}=.1-1 \mathrm{GeV}^{2}$ 
Parity-Violating Asymmetry Extrapolated to $\mathbf{Q}^{2}=\mathbf{0}$ (R.D. Young, R.D. Carlini, A.W. Thomas, and J. Roche, PRL 99, 122003 (2007))

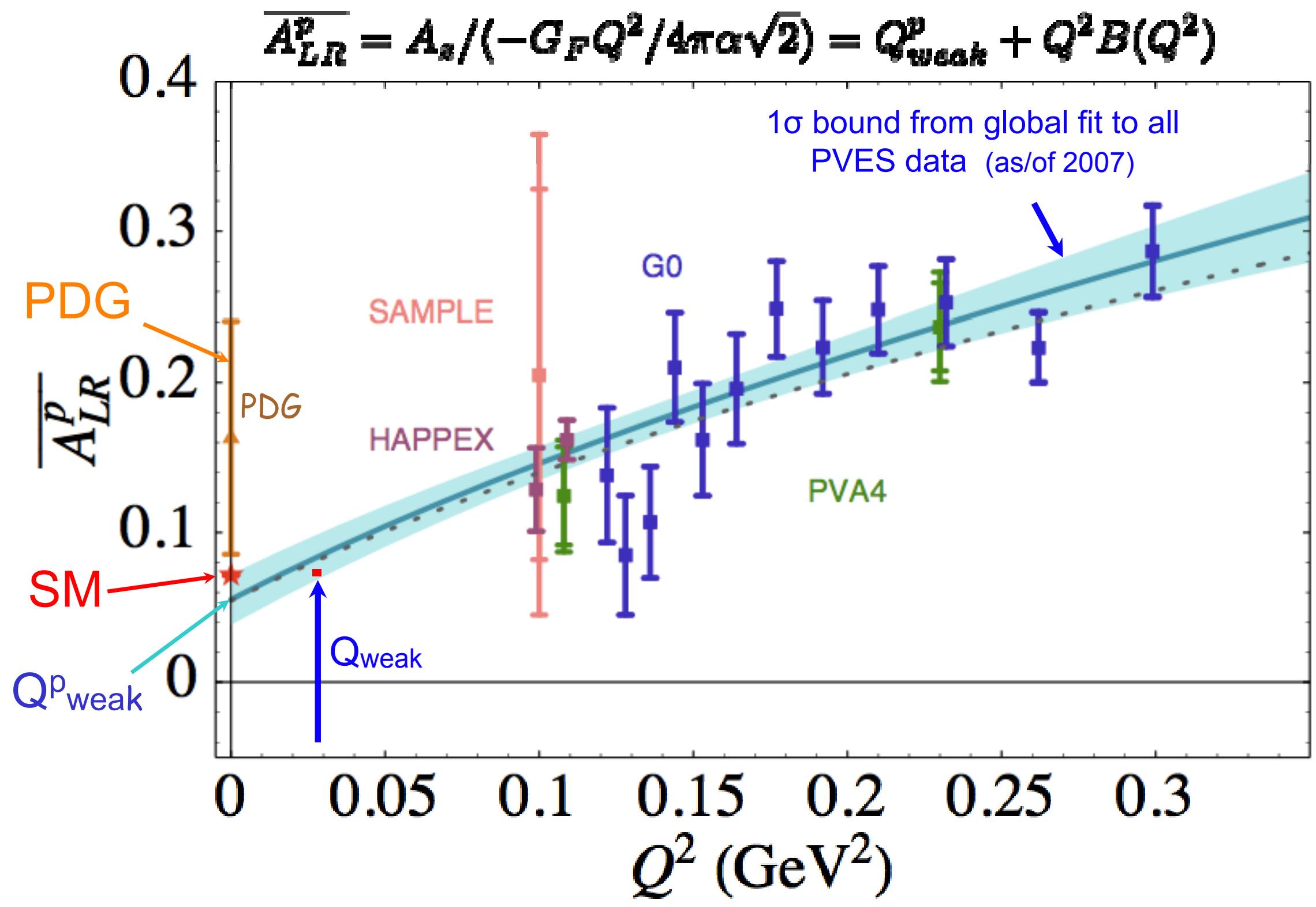




\section{Anticipated $Q^{p}$ Weak Uncertainties}

\begin{tabular}{|c|c|c|}
\hline & $\Delta \boldsymbol{A}_{\text {phys }} / \boldsymbol{A}_{\text {phys }}$ & $\Delta \mathbf{Q}_{\text {weak }}^{p} / \mathbf{Q}^{p}$ weak \\
\hline $\begin{array}{l}\text { Statistical ( } 2200 \text { hours production) } \\
\text { Systematic: }\end{array}$ & $2.1 \%$ & $3.2 \%$ \\
\hline $\begin{array}{l}\text { Hadronic structure uncertainties } \\
\text { Beam polarimetry } \\
\text { Absolute } Q^{2} \text { determination } \\
\text { Backgrounds } \\
\text { Helicity-correlated Beam Properties }\end{array}$ & $\begin{array}{l}-- \\
1.0 \% \\
0.5 \% \\
0.5 \% \\
0.5 \%\end{array}$ & $\begin{array}{l}1.5 \% \\
1.5 \% \\
1.0 \% \\
0.7 \% \\
0.7 \%\end{array}$ \\
\hline Total & $2.5 \%$ & $4.1 \%$ \\
\hline
\end{tabular}

$4 \%$ error on $Q^{p}{ }_{w}$ corresponds to $\sim 0.3 \%$ precision on $\sin ^{2} \theta_{W}$ at $Q^{2} \sim 0.03 \mathrm{GeV}^{2}$

$$
\begin{aligned}
Q_{W}(p)= & {\left[\rho_{N C}+\Delta_{e}\right]\left[1-4 \sin ^{2} \hat{\theta}_{W}(0)+\Delta_{e}^{\prime}\right] } \\
& +\square_{W W}+\square_{Z Z}+\square_{\gamma Z} .
\end{aligned}
$$

(Erler, Kurylov, Ramsey-Musolf, PRD 68, 016006 (2003))

$Q^{p}{ }_{w}=0.0713 \pm 0.0008$ theoretically

$1.1 \%$ error comes from $Q C D$ uncertainties in box graphs, etc. 


\section{Electroweak Radiative Corrections}

$$
\begin{aligned}
Q_{W}(p)= & {\left[\rho_{N C}+\Delta_{e}\right]\left[1-4 \sin ^{2} \hat{\theta}_{W}(0)+\Delta_{e}^{\prime}\right] } \\
& +\square_{W W}+\square_{z Z}+\square_{\gamma z} .
\end{aligned}
$$

\begin{tabular}{|c|c|c|c|c|}
\hline \multirow{2}{*}{\multicolumn{2}{|c|}{\begin{tabular}{|l|}
$Q^{p}$ Weak \\
$Q^{p}$ Weak \\
\end{tabular}}} & $\begin{array}{l}\text { Standard Model }\left(Q^{2}=0\right) \\
\text { experiment precision goal }\end{array}$ & 0.071 & \multirow[b]{2}{*}{$\begin{array}{l}\text { Erler, Kurylov, } \\
\text { Ramsey-Muslolf, } \\
\text { PRD 68(2003)016006 }\end{array}$} \\
\hline & & $\begin{array}{l}\text { Source } \\
\Delta \sin \theta_{W}\left(M_{Z}\right) \\
Z \gamma \text { box } \\
\Delta \sin \theta_{W}(Q)_{\text {hadronic }} \\
W W, Z Z \text { box - pQCD } \\
\text { Charge symmetry } \\
\text { Total }\end{array}$ & $\begin{array}{l}\text { Uncertainty } \\
\quad \pm 0.0006 \\
\pm 0.0005 \\
\pm 0.0003 \\
\pm 0.0001 \\
0 \\
\pm 0.0008\end{array}$ & \\
\hline & $\begin{array}{r}\text { Estim } \\
\text { TBE } \\
\text { TBE }\end{array}$ & $\begin{array}{l}\text { ates of } \gamma \text {-Z box diagrams on } A_{P} \\
\text { (Tjon, Blunden, Melnitchouk) } \\
\text { arXiv:0903.2759 } \\
\text { (Gorchtein \& Horowitz) } \\
\text { hys. Rev. Lett. 102, } 091806 \text { (2009) }\end{array}$ & $\begin{array}{l}\text {, at Qweak Kir } \\
0.13 \% \text { (hadro } \\
\sim 6 \% \text { (disper }\end{array}$ & $\begin{array}{l}\text { matics } \\
: \mathrm{N} \text { and } \Delta \text { ) } \\
\text { in relations; PVDIS FF) }\end{array}$ \\
\hline
\end{tabular}




\section{Energy Scale of an "Indirect" Search for New Physics}

- Parameterize New Physics contributions in electron-quark Lagrangian

$$
\mathrm{L}_{\mathrm{e}-\mathrm{q}}^{\mathrm{PV}}=\mathrm{L}_{\mathrm{SM}}^{\mathrm{PV}}+\mathrm{L}_{\mathrm{NEW}}^{\mathrm{PV}}=-\frac{G_{F}}{\sqrt{2}} \bar{e} \gamma_{\mu} \gamma_{5} e \sum_{q} C_{1 q} \bar{q} \gamma^{\mu} q+\frac{g^{2}}{4 \Lambda^{2}} \bar{e} \gamma_{\mu} \gamma_{5} e \sum_{q} h_{V}^{q} \bar{q} \gamma^{\mu} q
$$

- A 4\% Q Weak $_{\text {weasurement probes with }}$ $95 \%$ confidence level for new physics

$g$ : coupling constant, $\Lambda$ : mass scale

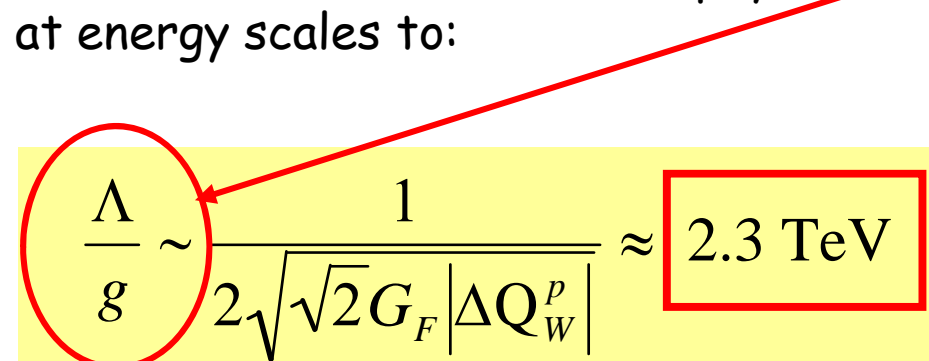

- If LHC uncovers new physics, then precision low $Q^{2}$ measurements will be needed to determine charges, coupling constants, etc.

Some current and proposed mass scale $\Lambda(\mathrm{TeV})$ lower limits

\begin{tabular}{|c|cc|ccc|cc|}
\hline & \multicolumn{2}{|c|}{$Z^{\prime}$ models } & \multicolumn{2}{|c|}{ leptoquark } & \multicolumn{2}{c|}{ compositeness } \\
& $m\left(Z_{\chi}\right)$ & $m\left(Z_{L R}\right)$ & $m_{L Q}($ up $)$ & $m_{L Q}($ down $)$ & $e-q$ & $e-e$ \\
\hline Current direct search limits & 0.69 & 0.63 & 0.3 & 0.3 & - & - \\
Current electroweak fit & 0.78 & 0.86 & 1.5 & 1.5 & $11-26$ & $8-10$ \\
$0.6 \% Q_{W}(\mathrm{Cs})$ & 1.2 & 1.3 & 5.1 & 5.4 & 28 & - \\
$13.1 \% Q_{W}(e)$ & 0.66 & 0.34 & - & - & - & 13 \\
$4 \% Q_{W}(p)$ & 0.95 & 0.45 & 6.5 & 4.6 & 28 & - \\
\hline \hline
\end{tabular}




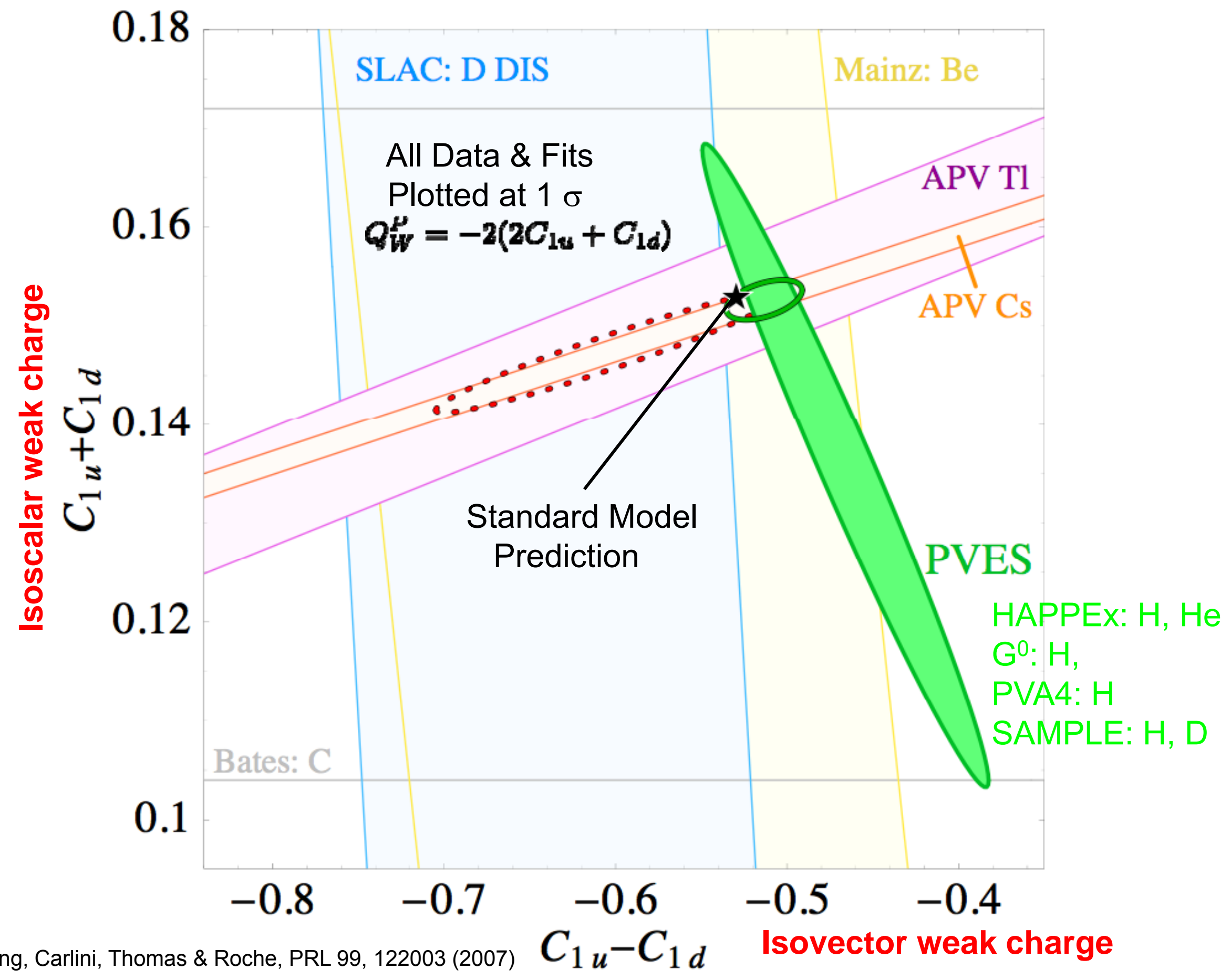




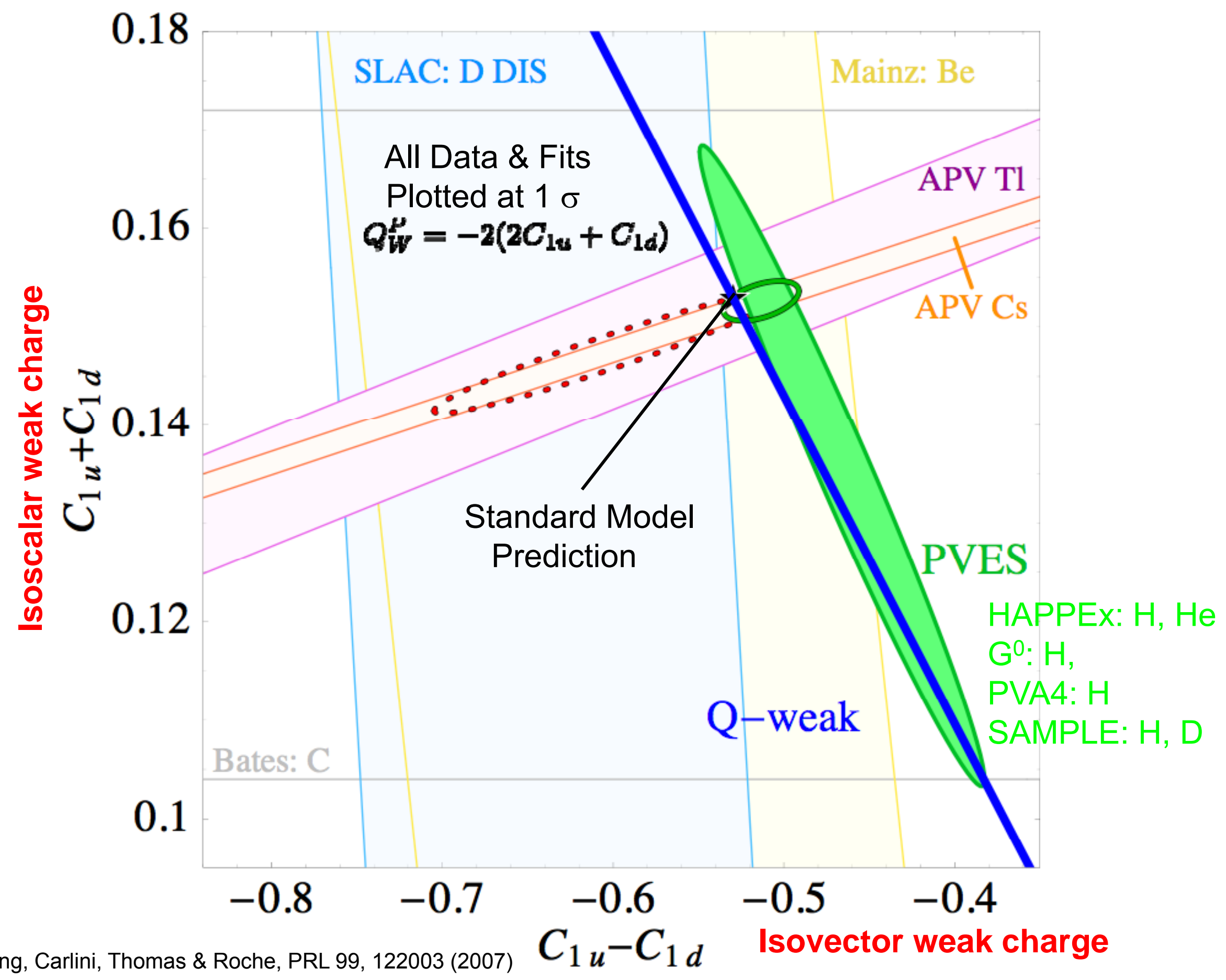




\section{New Physics Reach}

$$
\begin{aligned}
& \mathcal{L}_{\mathrm{SM}}^{\mathrm{PV}}=-\frac{G_{F}}{\sqrt{2}} \bar{e} \gamma_{\mu} \gamma_{5} e \sum_{q} C_{1 q}^{\mathrm{SM}} \bar{q} \gamma^{\mu} q \\
& \mathcal{L}_{\mathrm{NP}}^{\mathrm{PV}}=-\frac{g^{2}}{4 \Lambda^{2}} \bar{e} \gamma_{\mu} \gamma_{5} e \sum_{q} h_{V}^{q} \bar{q} \gamma^{\mu} q \underbrace{\text { PRD6(2003) }}_{\text {Erler Kurylov, Ramsey-Musolf., }}
\end{aligned}
$$

Arbitrary quark flavour dependence of new physics:

$$
h_{V}^{u}=\cos \theta_{h} \quad h_{V}^{d}=\sin \theta_{h}
$$

Data sets limits on: $\frac{\boldsymbol{g}^{2}}{\Lambda^{2}} \quad \begin{gathered}\text { Young, Carlini, Thomas \& Roche, PRL 99, } \\ 122003(2007)\end{gathered}$ 


\section{Lower Bound for "Parity Violating” New Physics}

10
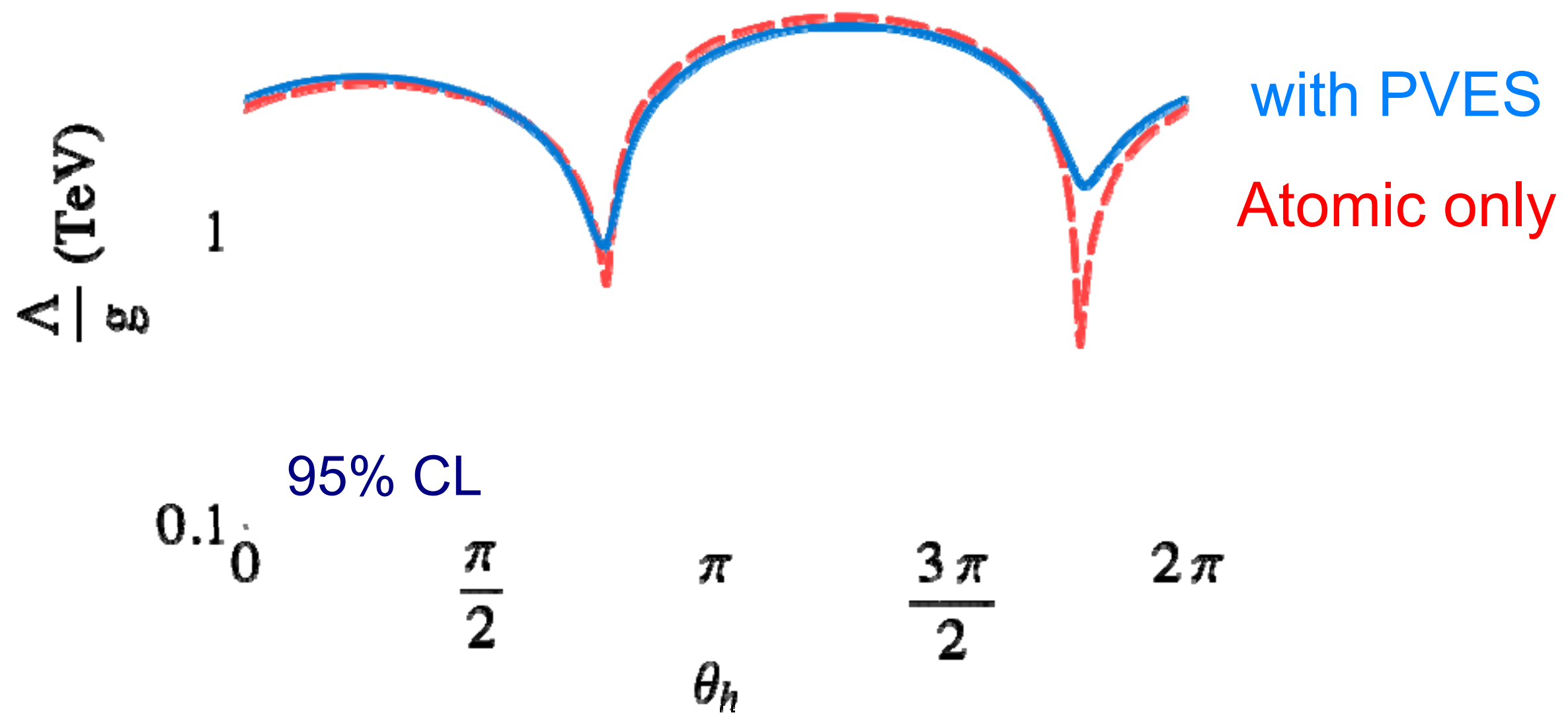

New PV physics scale > 0.9 TeV! (from 0.4 TeV) 


\section{Lower Bound for “Parity Violating” New Physics}

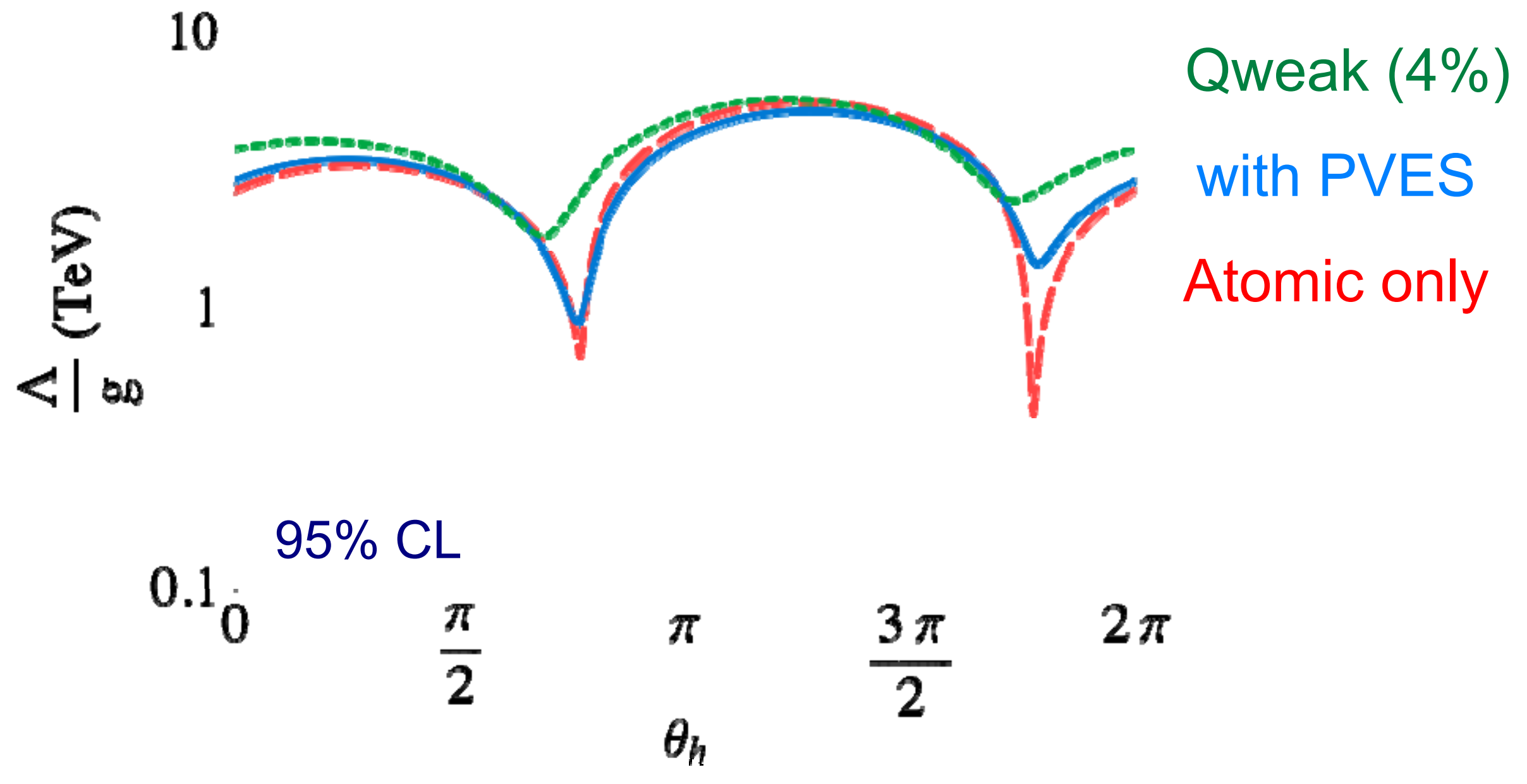

Qweak constrains new PV physics to beyond $2 \mathrm{TeV}$

Young, Carlini, Thomas \& Roche, PRL 99, 122003 (2007) 
$Q_{\text {weak }} \& Q_{\text {weak }}^{e}$ - Complementary Diagnostics for New Physics

\section{$\underline{\text { JLab Qweak }}$}

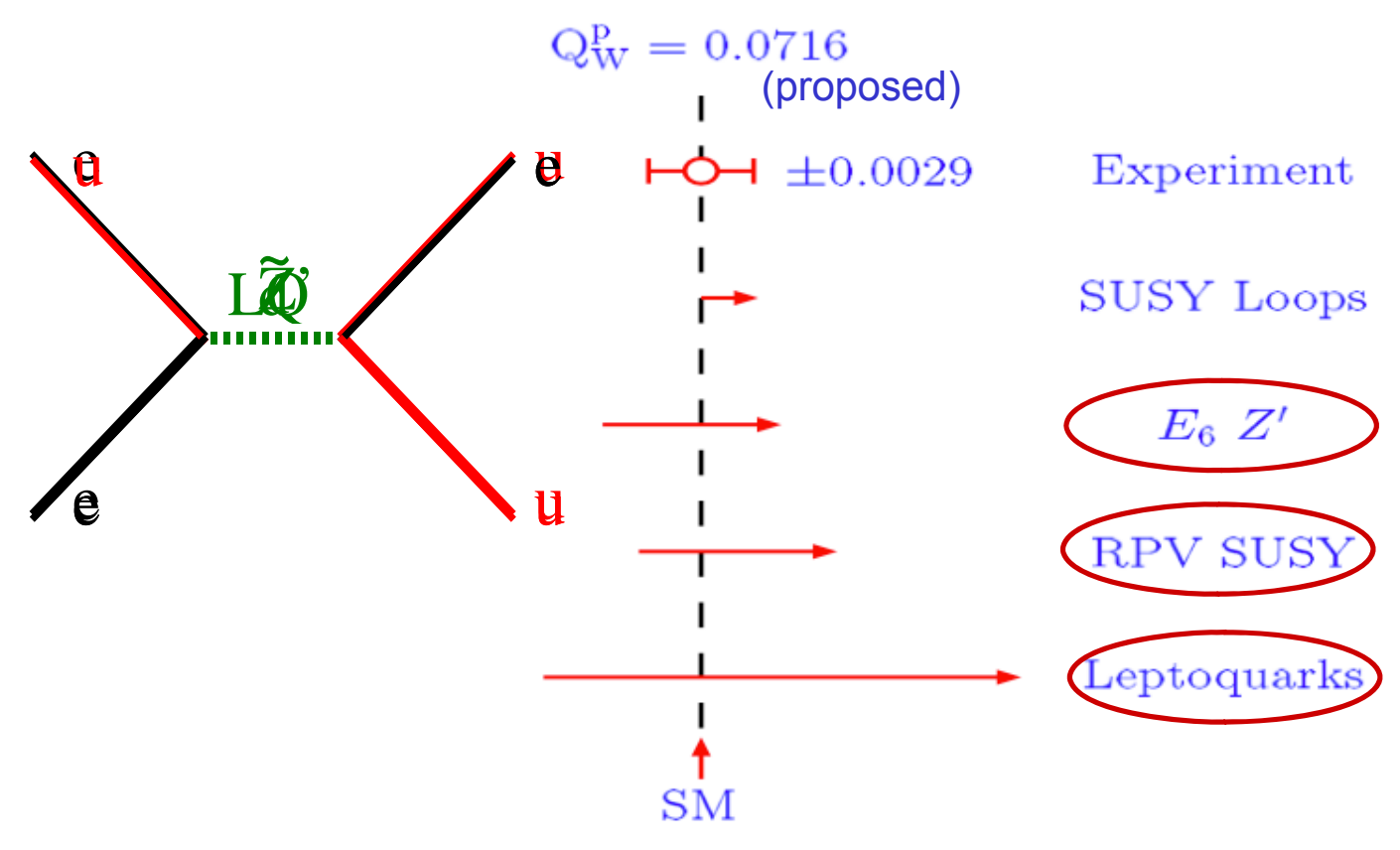

Erler, Kurylov, Ramsey-Musolf, PRD 68, 016006 (2003)

- arrows show allowed "pull" of weak charges by new physics as constrained by previous experiments

- electron and proton weak charge experiments are complementary

\section{SLAC E158}

$$
{ }^{-} \mathrm{Q}_{\mathrm{W}}^{\mathrm{e}}=0.0449
$$

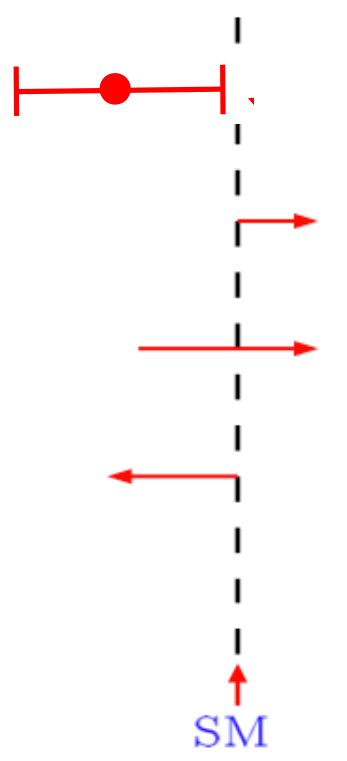




\section{Relative Shifts in Proton and Electron Weak Charges due to SUSY Effects}

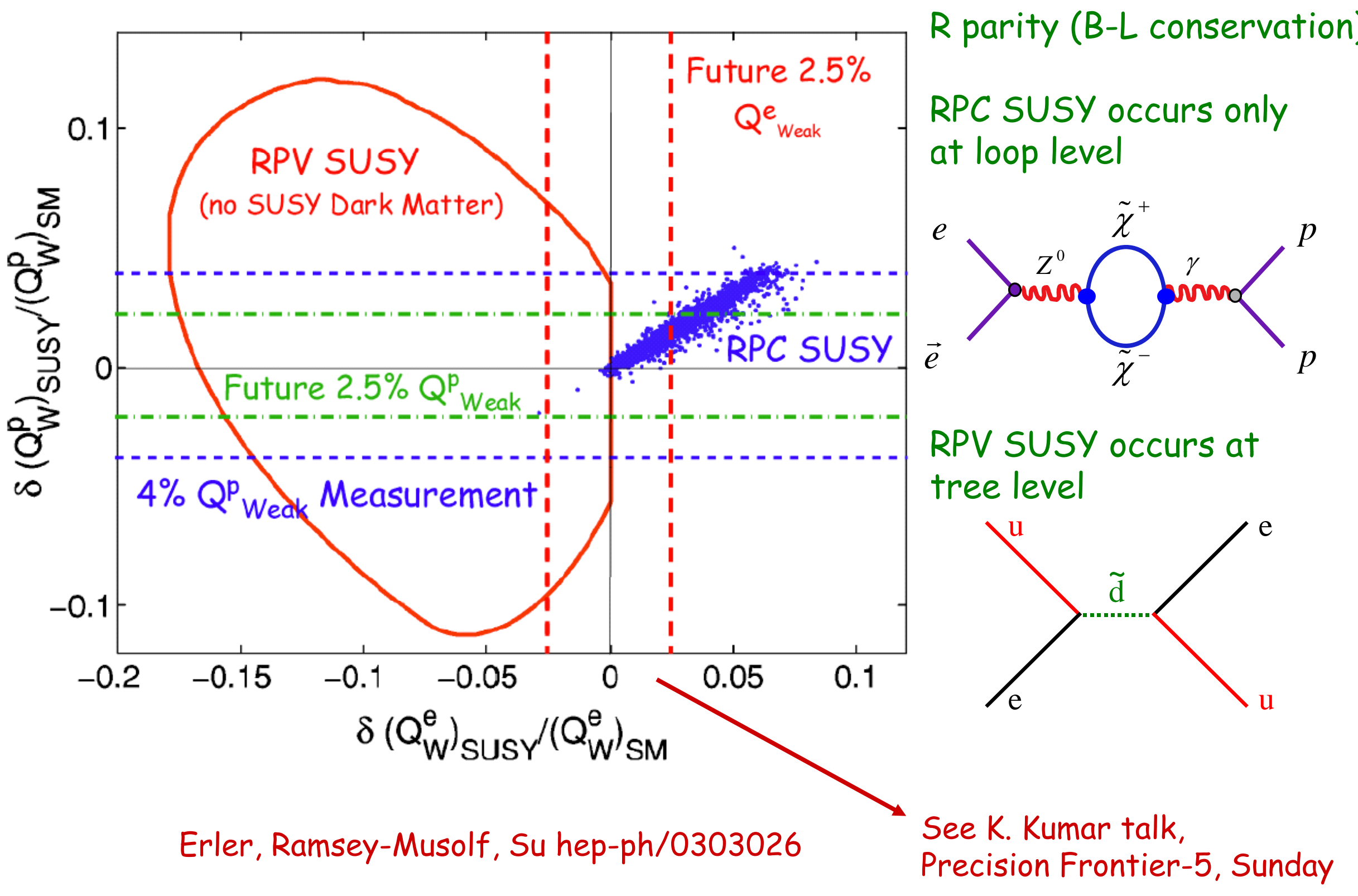




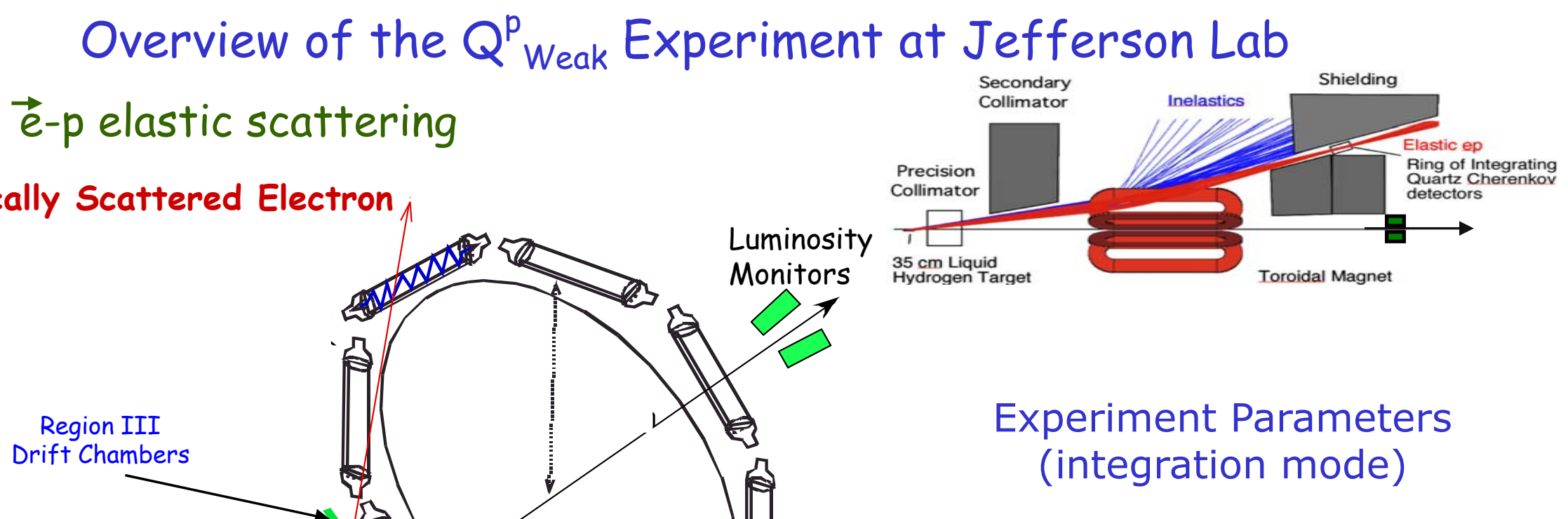

Elastically Scattered Electron

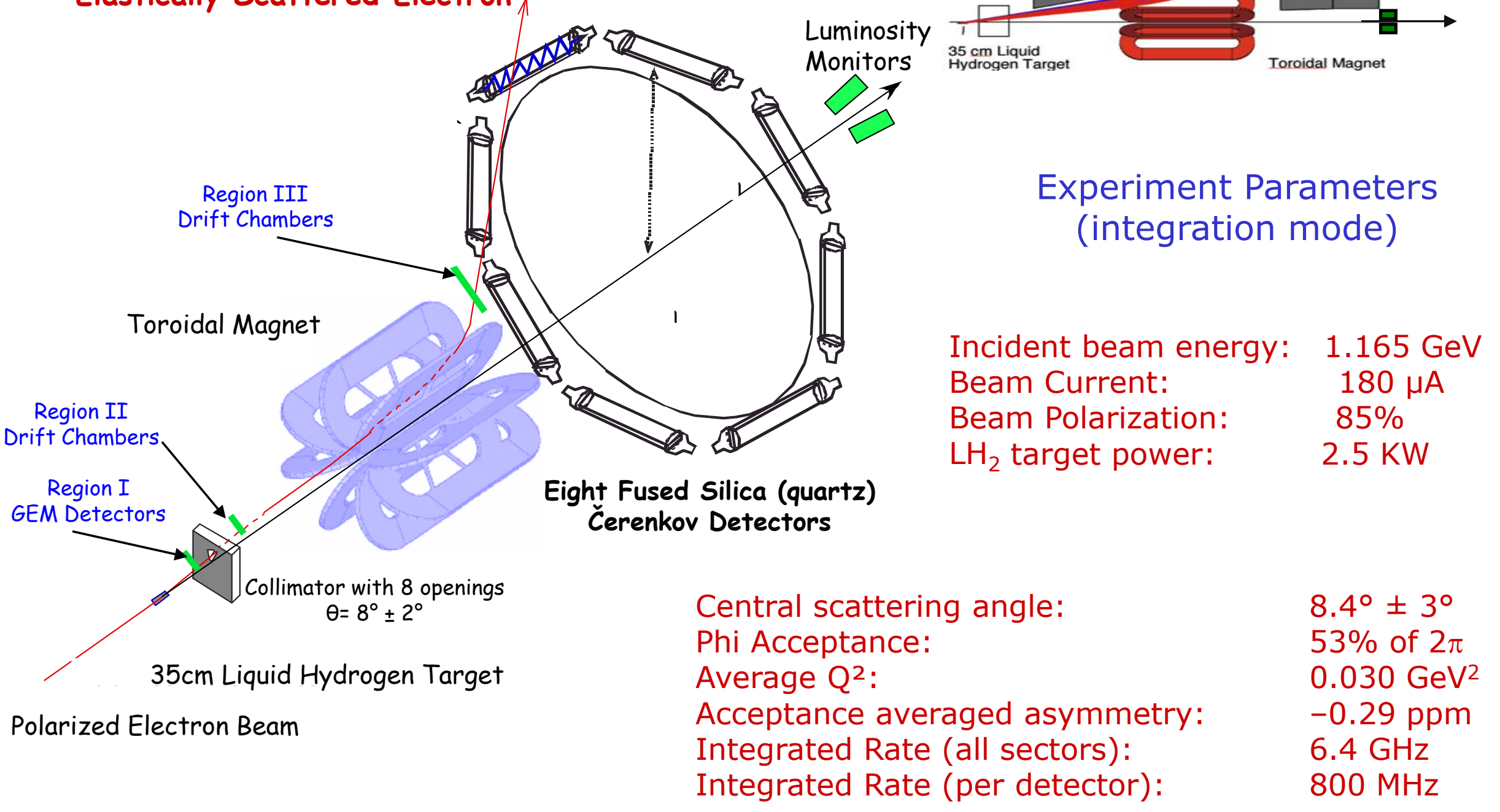




\section{Qweak overview}

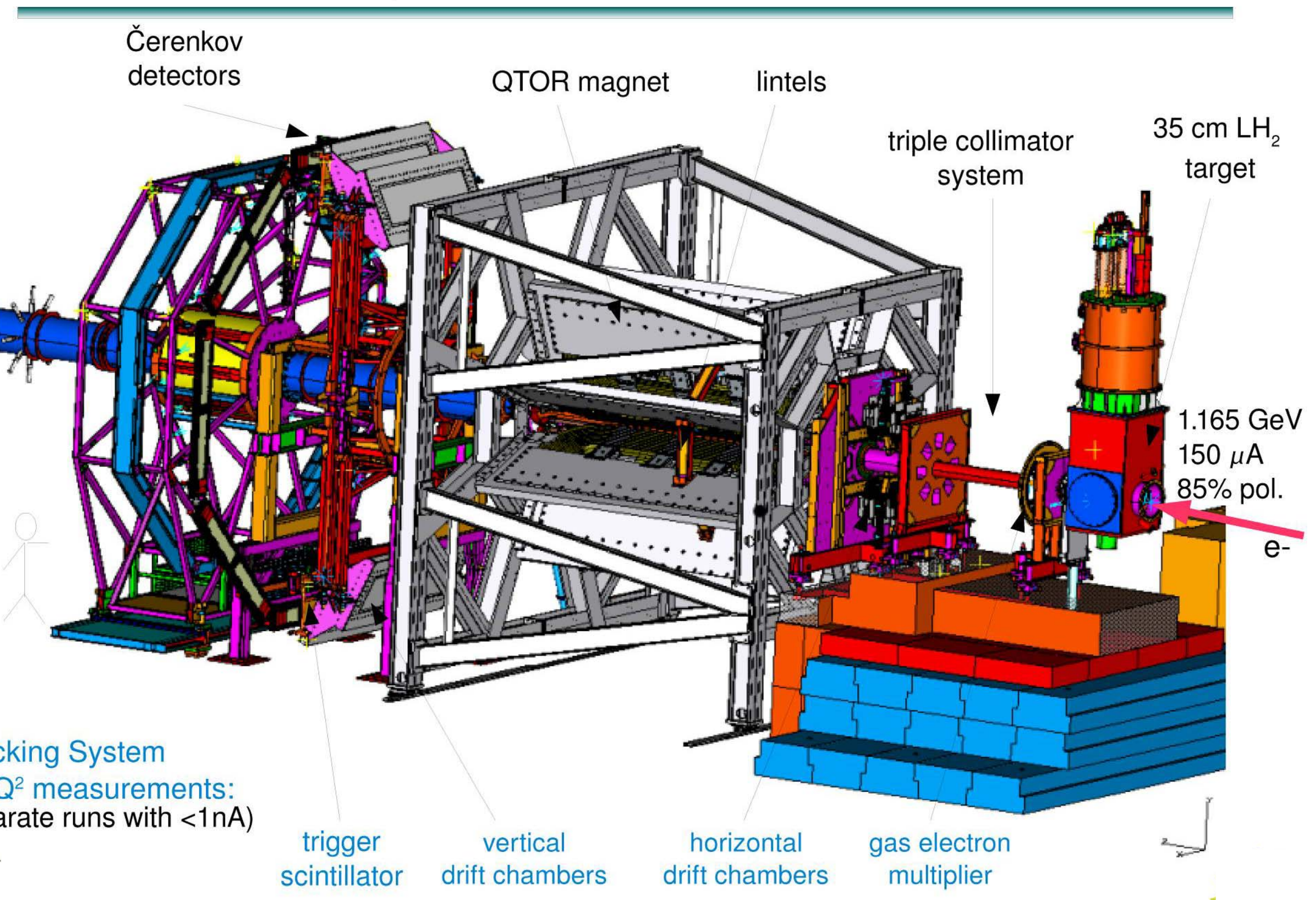




\section{$Q_{\text {Weak }}^{p}$ Status of Production Mode Components}

QTOR: Toroidal Magnet

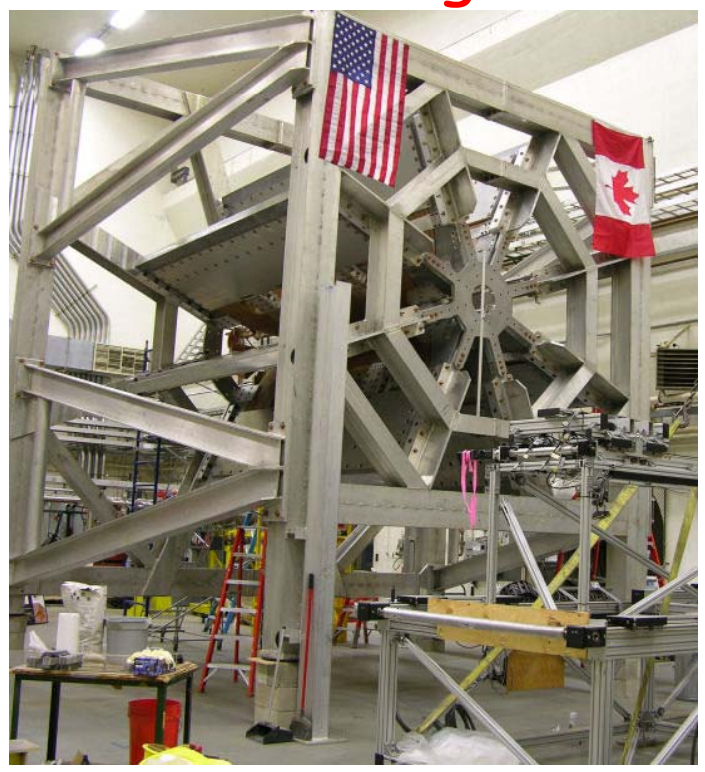

Main Quartz Detector and Electronics

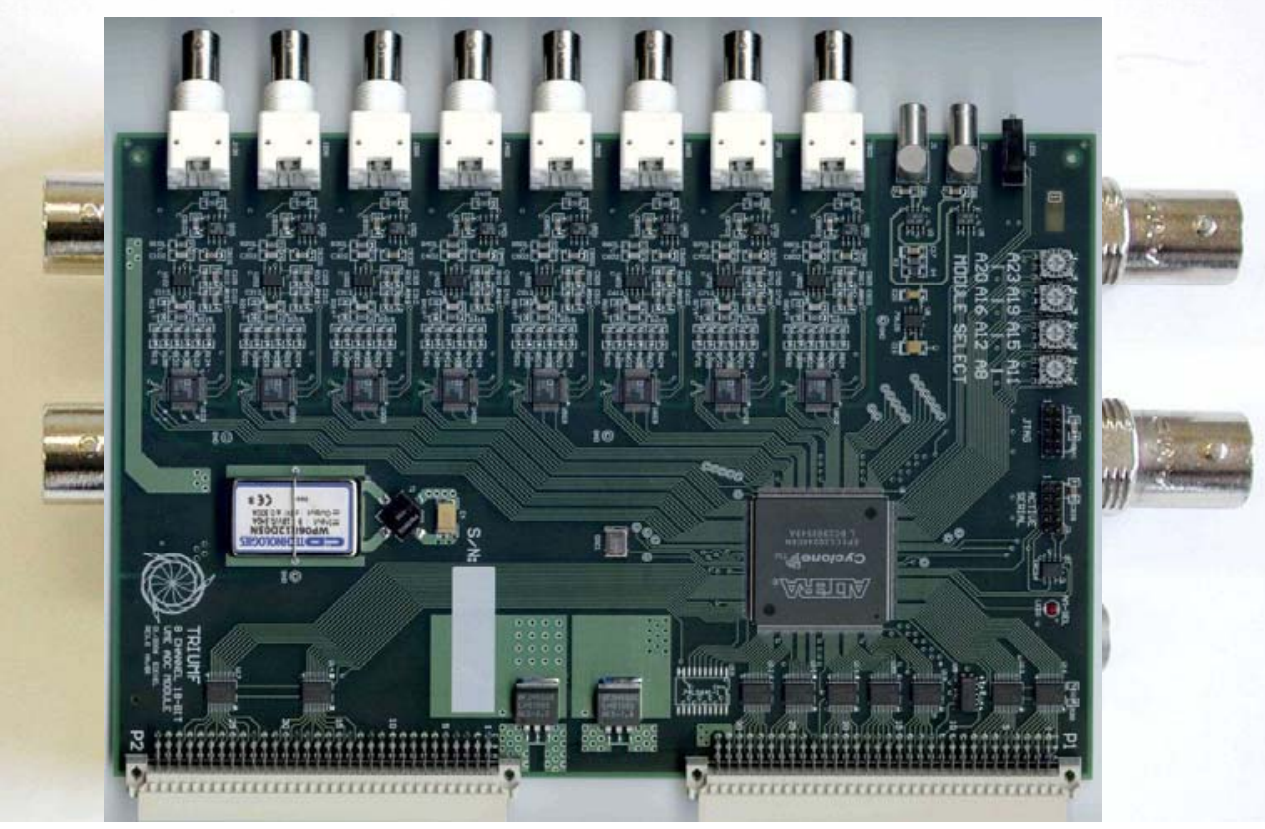

High Power Cryogenic Target

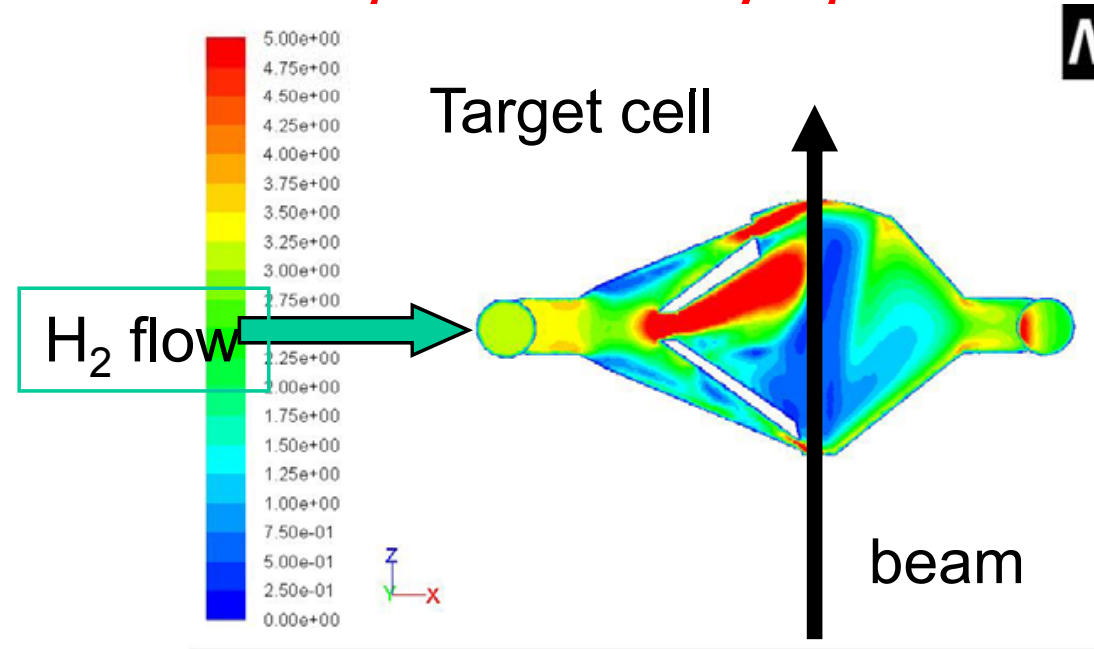

Contours of Velocity Magnitude $(\mathrm{m} / \mathrm{s})$

Nov 17, 2008
FLUENT 12.0 (3d, pbns, rke)

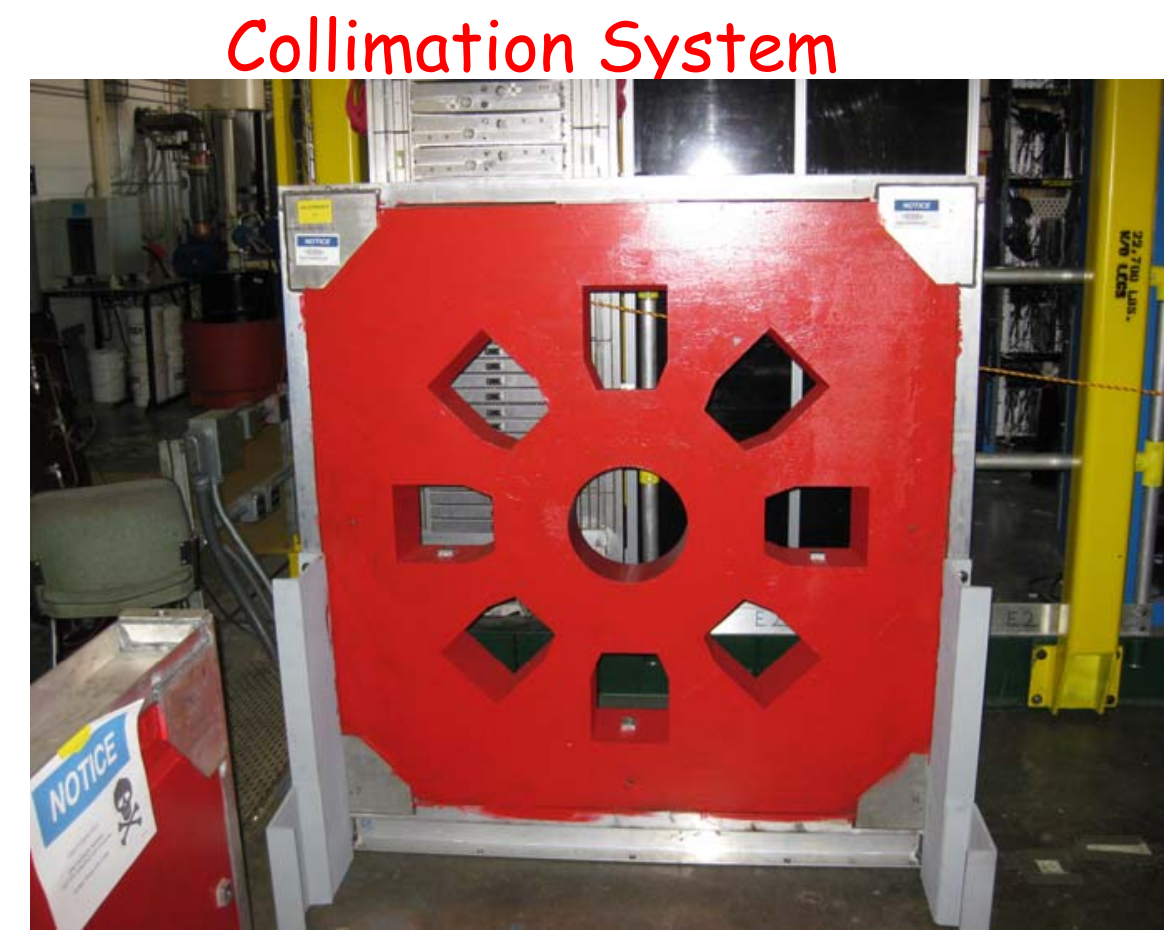




\section{$Q_{\text {Weak }}^{p}$ - Status of Calibration Mode Components}

The $Q^{2}$ of the experiment is calibrated at low beam current with a tracking system.

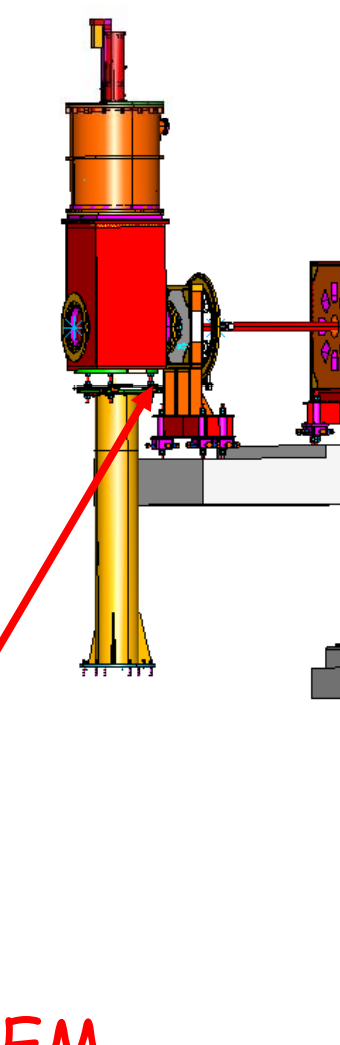

Region 1: GEM
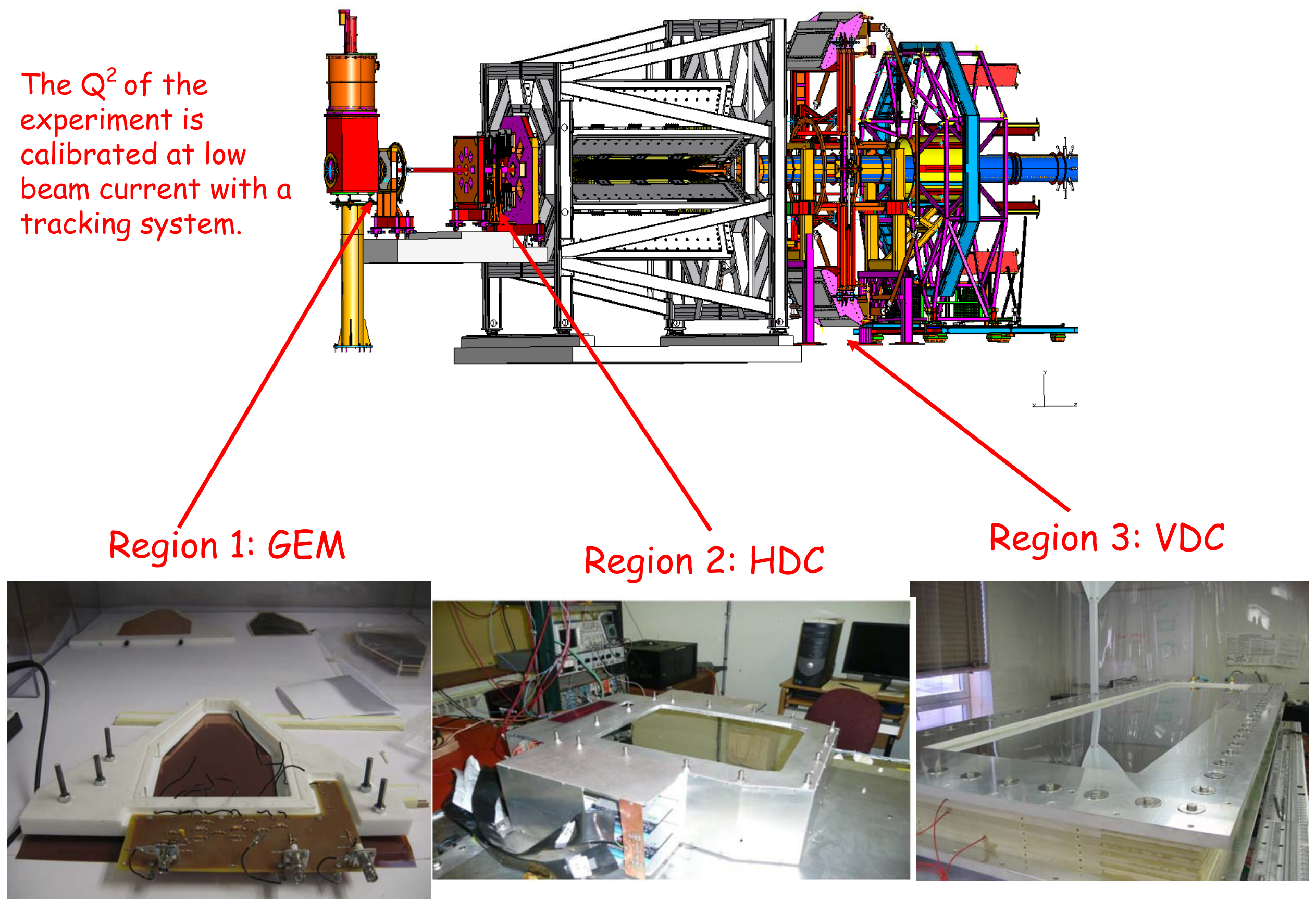


\section{Schedule and Summary}

$Q_{\text {weak }}^{p}$ experiment schedule

- Installation begins in October 2009

- Commissioning/Engineering run May 2010 - July 2010

- First production run: September 2010 - April 2011

- Second production run: November 2011 - May 2012

- Beamtime end = $12 \mathrm{GeV}$ upgrade shutdown in May 2012 (last experiment to run in Hall $C$ before shutdown)

\section{A successful $Q_{\text {Weak }}^{\mathcal{P}}$ experiment will provide:}

- Precision measurement of the proton's weak charge in the simplest system.

- Sensitive search for new physics with CL of 95\% at the 2.3 TeV scale.

- Fundamental $10 \sigma$ measurement of the running of $\sin ^{2} \theta_{w}$ at low energy. 